\title{
Regenerative Biology of Tendon: Mechanisms for Renewal and Repair
}

\author{
Nathaniel A. Dyment ${ }^{1}$ • Jenna L. Galloway ${ }^{2,3}$
}

Published online: 4 July 2015

(C) Springer International Publishing AG 2015

\begin{abstract}
Understanding the molecular and cellular mechanisms underlying tissue turnover and repair are essential towards addressing pathologies in aging, injury, and disease. Each tissue has distinct means of maintaining homeostasis and healing after injury. For some, resident stem cell populations mediate both of these processes. These stem cells, by definition, are self-renewing and give rise to all the differentiated cells of that tissue. However, not all organs fit with this traditional stem cell model of regeneration, and some do not appear to harbor somatic stem or progenitor cells capable of multilineage in vivo reconstitution. Despite recent progress in tendon progenitor cell research, our current knowledge of the mechanisms regulating tendon cell homeostasis and injury response is limited. Understanding the role of resident tendon cell populations is of great importance for regenerative medicine-based approaches to tendon injuries and disease. The goal of this review is to bring to light our current knowledge regarding tendon progenitor cells and their role in tissue
\end{abstract}

This article is part of the Topical Collection on Molecular Biology of Adult Stem Cells

Nathaniel A. Dyment

dyment@uchc.edu

Jenna L. Galloway

jgalloway1@mgh.harvard.edu

1 Center for Regenerative Medicine and Skeletal Development, Department of Reconstructive Sciences, School of Dental Medicine, University of Connecticut Health Center, 263 Farmington Ave, Farmington, CT 06030, USA

2 Center for Regenerative Medicine, Department of Orthopaedic Surgery, Massachusetts General Hospital, Harvard Medical School, 185 Cambridge Street, Boston, MA 02114, USA

3 Harvard Stem Cell Institute, Cambridge 02138, MA, USA maintenance and repair. We will focus on pressing questions in the field and the new tools, including model systems, available to address them.

Keywords Tendon · Tendon injury · Tendon maintenance · Tendon repair $\cdot$ Progenitor cells

\section{Introduction}

Tendons are a vital component of our musculoskeletal system. They transmit force from the muscles to the bones, permitting movement. Adult tendons are primarily composed of highly ordered type I collagen molecules and are surrounded by a paratenon or sheath, which reduces friction, allowing the tendon to move smoothly among its neighboring tissues $[1,2]$. Tendons are highly prone to injury and it is estimated that millions of injuries occur each year, resulting in significant healthcare costs [3]. Their limited healing response poses significant clinical problems as traditional repair strategies often lead to inconsistent and undesirable outcomes [4]. After injury, tendons heal through scarring and have altered biomechanical properties, ultimately affecting their function [5-7]. One of the largest risk factors for tendon injury is aging, which is characterized by altered mechanical properties and slower healing responses $[8-10,11 \bullet \bullet]$.

During vertebrate development, tendons form in all anatomic locations where muscle connects bone. Their embryological origins are distinct based on their position: cranial tendons are neural crest derived, axial tendons form from the paraxial somitic mesoderm, and the limb tendons arise from the lateral plate mesoderm [12-14]. Scleraxis, a basic helix loop helix transcription factor, is the earliest marker of tendon progenitors in all anatomic locations and is required for the normal formation of the limb force producing tendons [15]. In 
the limb, the tendon primordia form in several locations near the condensing cartilage structures and eventually connect the muscle and bone. As they organize and differentiate among the forming musculoskeletal system, they express the transcription factors Egrl, Egr2 (Lejard, 2011) and Mohawk $(M k x)[16,17]$, and tendon matrix components Tenomodulin (Tnmd) $[18,19]$ and type I collagen [20]. During these later embryonic and early postnatal stages, a rich collagen matrix is deposited and what began as a cellular structure transitions to a tissue primarily composed of extracellular matrix.

After birth, there are several changes to tendon cells and their surrounding extracellular matrix. Axially arranged collagen fibrils of the tendons grow in both length and diameter $[21-23,24 \cdot]$. Cells within the tendon also shift in their morphology and number. Young tendons have many rounded cells that have been referred to as tenoblasts, while adult and aged tendons have fewer cells that are long and thin $[11 \bullet \cdot, 25]$. The elongated cells have been referred to as tenocytes or internal tendon fibroblasts and are less metabolically active than tenoblasts [26]. Recent studies of tail tendons using 3D electron microscopy have shown that cell body length decreases, but the surface area of tendon cells increases during postnatal growth stages [24•]. These cell shape changes occur while the cell-cell contacts are maintained and the collagen fibrils are increasing in diameter and length [24•]. This ultimately causes the internal tendon cells to adopt a stellate appearance and support the notion that tenoblasts become tenocytes as the animal ages [27]. Other studies have suggested that tenocytes can revert to tenoblasts following injury [28, 29]. However, due to a paucity of cell type specific expression markers and lineage studies, the definitive relationship between these cell populations during development and injury is unresolved.

Adult tendon structure is highly ordered, consisting of a hierarchy of collagen fibrils and fibers that form tendon fascicles with loose connective tissue between and around the fascicles. The tenocytes that reside within the collagen fascicles are thought to be a relatively homogeneous population of elongated cells with a high nuclear to cytoplasmic ratio $[26,30,31]$. Unlike the internal population, the loose connective tissue surrounding the collagen fascicles contains a more heterogeneous mix of vascular, nerve, and mesenchymal cells $[4,11 \bullet, 30,32]$. The mesenchymal cells or fibroblasts within the loose connective tissue are defined by the anatomical location within the tendon just as tenocytes are defined based on their location within the tendon fascicles. However, the origins of cell populations in the surrounding loose connective tissue are not fully elucidated and further research is needed to understand the function and contribution of these cells to tissue maintenance, growth, and repair.

\section{Tendon Maintenance}

Tissue homeostasis is driven by a combination of cell and matrix turnover to maintain normal steady state conditions. Tendons are thought to have very low turnover, which is hypothesized to limit their healing potential. Methods to measure tendon turnover have yielded halflife values ranging from 2 months to 200 years and can depend on the matrix molecule being analyzed [33-35]. A recent study utilizing nuclear bomb testing in the midtwentieth century as a means to examine tendon turnover in human populations gave more evidence for limited tissue renewal $[36 \cdot \bullet]$. The study concluded that Achilles tendons from people born before, during, and after the nuclear testing had very limited tissue turnover in adulthood. In fact, the ${ }^{14} \mathrm{C}$ content in their tendons correlated with the atmospheric concentration during growth $(0$ 17 years of age), suggesting that collagen synthesis occurs primarily during this time frame and there is not significant tissue turnover at later stages of life. However, their findings describe the turnover for the entire tissue, containing extracellular and cellular components. The authors speculate that their findings cannot exclude the possibility of higher turnover in a small population of cycling cells or matrix molecules. Nevertheless, traditional methods to measure cell proliferation in rodents, including injections of thymidine analogs (e.g., BrdU, EdU) and immunostaining for cell proliferation markers (e.g., Ki-67 and PCNA), have revealed low cell proliferation within the internal fibroblast population during postnatal time points $[3,6$, $19,37,38]$, and with higher labeling within the paratenon $[3,19,39]$. The limited number of proliferative cells within the tendon at early postnatal stages is surprising as tendons are in an active state of growth. Although much of this growth has been attributed to expansion of the extracellular matrix, it has been suggested that cells proliferate within their channels along the tendon's longitudinal axis during these periods [24•]. Therefore, it may be necessary to utilize methods to detect small differences in turnover and proliferation rates, such as the doxycyclineinducible H2B-GFP reporter mouse [40-43], which has been shown to be much more sensitive than BrdU or EdU labeling, and multicolor Cre reporter mice (e.g., R26R-Confetti and R26R-Rainbow) [44-46], which allow for analysis of clonal expansion without the necessity of BrdU or EdU incorporation during S-phase. These new tools may still reveal low cell proliferation rates compared to other tissues, but they will also provide greater insight with higher accuracy into the cellular dynamics of tendon cells during growth and maintenance.

Although tendons appear to have modest turnover, they are sensitive to mechanical stimulation and react to changes in physical activity [47, 48]. During exercise, tendon cells 
become metabolically active, which corresponds with increases in tendon mass, cell density, and cross sectional area $[49,50]$. Exercise also augments cell proliferation, as identified by BrdU labeling in specific regions of the tendon near muscle and bone attachments [39]. However, co-expression studies are necessary to determine which specific cell populations are dividing and responding to changes in physical activity. In general, exercise appears to have a positive effect on tendon characteristics with increases in expression of tendonassociated genes, Scx, Tnmd, and Colla2 [49], and proliferation and collagen production capacities in culture (Zhang, 2010). Exercise also stimulates collagen production in human tendons, increasing by $6 \mathrm{~h}$ and peaking at $24 \mathrm{~h}$ after activity $[34,51]$. In contrast, overloading or unloading the tendon results in pathological degenerative changes to the tendon tissue [52-54]. In both scenarios, there is upregulation of matrix degrading factors and a shift in the distribution of matrix components $[55,56]$. Some studies have indicated that the change in matrix is due to altered cell metabolism rather than explicit gene expression changes [57, 58]. However, other studies have demonstrated increases in insulin-like growth factor 1 (IGF-1) and in cartilage program gene expression during overuse conditions $[59,60]$, and this may underlie the metabolic and extracellular matrix changes that are observed. Nevertheless, the tendon cells appear to be active participants in the regulation and maintenance of tendon tissue in response to changes in physical activity.

Aging is a significant contributor to tendon degeneration and injury. The pathogenesis of this process is not well understood, and many groups have investigated the matrix, mechanical, and cellular changes that occur with aging. As an animal matures, tendon tensile strength increases and healing capacity diminishes [61-64]. Increased tendon stiffness is reported between children and adults [65], and in aging, increases and decreases in stiffness have been observed [66, 67]. Cellular aging can involve genetic, epigenetic, metabolic, and proliferative changes as well as senescence and stem cell exhaustion [68-70]. Studies have shown that aged tendons have fewer cells, decreased collagen fiber alignment, and altered extracellular matrix $[10,11 \bullet \bullet, 25,71]$. Cultured progenitor cell populations from aged tendons display reduced proliferation, altered differentiation capacities, and increased expression of senescence associated genes such as $\mathrm{p} 14^{\mathrm{ARF}}$ and $\mathrm{p} 16^{\mathrm{INK} 4 \mathrm{~A}}$ $[68,72]$. Recent research also described a role for microRNAs in regulating tendon cell senescence in cell culture [73]. However, several questions remain regarding the underlying cause of the age-related degenerative changes and which changes initiate the decline in the tissue. A better mechanistic understanding of the native cellular processes that contribute to tendon aging is necessary to address the end-stage degenerative phenotype seen by clinicians. Moving forward, in vivo models of aging coupled with genetic lineage and conditional loss of function studies can be employed to better understand the molecular changes that occur to resident cell populations with age.

\section{Injury Repair Mechanisms}

Each tissue has distinct modes of regulation for their recovery and repair following injury. In the classical stem cell hierarchical model, a stem cell activates following injury, proliferates, and differentiates to make multiple cell types [42, 45, 74]. The skin and intestine are classical examples of such a hierarchy and have been excellent model systems to address many questions in regenerative biology. Within each of these systems, the stem cells reside in a distinct location termed a niche, which can regulate the maintenance and activity of stem cell populations [75]. Other tissues, however, utilize different mechanisms for repair. Specialized cells of the liver and pancreas can divide and replenish lost cell types after injury, but stem cells capable of multilineage repopulation have not been identified for these tissues [76, 77]. In contrast, organs such as the heart have very limited repair abilities. The existence of stem cells that maintain and repair these tissues is controversial, and differentiated cell populations are thought to be primarily responsible for the limited proliferation and repair that does occur [78]. In addition, injury can often result in tissue fibrosis, which can impede organ function $[79,80]$. In the tendon, we understand very little about the cellular mechanisms governing the repair process. In efforts to identify tendon stem cells in the adult tendon, cells from human and mouse tendons were isolated in culture, clonally expanded, and characterized. These cells express subsets of mesenchymal stem cell markers, retain multilineage differentiation potential, and can be clonally and serially propagated to form tendon-like tissue after transplantation [11••, 70, 81, 82]. Although these populations display potent progenitor characteristics in culture, we know much less about the origin, identity, and activity of these cells during homeostasis and injury in vivo.

In understanding the identity of the cells involved in the injury response, it is important to focus on the anatomy of the tendon itself (see Fig. 1). The cell populations contributing to repair have been described to arise from intrinsic and extrinsic origins [83-90], but the true contribution of each population to the healing response has not been clearly defined. Intrinsic cells are typically defined as the tenocytes or internal fibroblasts residing within the collagen fascicles (Fig. 1, green cells) [30]. The extrinsic cell populations include cells within the loose connective tissue surrounding the tendon (epitenon and paratenon), within the outer tendon sheath, or neighboring blood vessels (i.e., perivascular cells) and inflammatory cells (Fig. 1, red and black cells) [4, 11••, 91]. Extrinsic cell populations are thought to be the first mesenchymal cell populations to respond during the inflammatory phase of the healing 


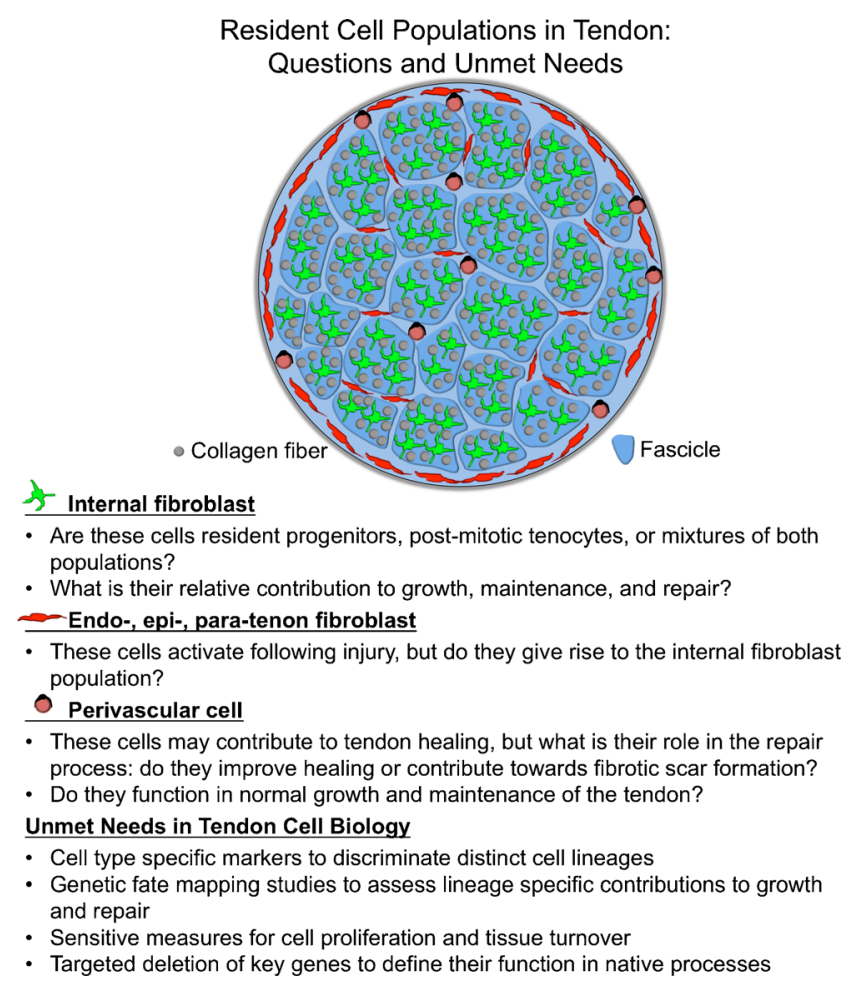

Fig. 1 Research questions and unmet needs related to the biology of resident cell populations in tendon. Several mesenchymal cell types have been identified based on their anatomical location within and around the tendon. These cell types include the following: (1) the internal tendon fibroblasts (green) situated between collagen fibers within the tendon fascicles, (2) the endo-, epi-, and paratenon fibroblasts $(\mathrm{red})$ found within the loose connective tissue surrounding the collagen fascicles, and (3) the perivascular cells (black) surrounding vessels in and around the tendon. While identifying these populations based on their location is clear, we have less understanding of the phenotypical differences between these cell populations and the markers to discriminate between them. In this figure, we have provided a schematic for the anatomical positions of these cell populations. We have also outlined the pertinent research questions related to each population and the unmet needs that should be addressed in the tendon cell biology field moving forward

response $[4,6,92]$. During later stages of repair and remodeling, it has been suggested that intrinsic cell populations participate in the healing process and produce larger and more aligned collagen fibers [4]. Because of the lack of biological tools to genetically label the appropriate cell populations and perform detailed lineage tracing analysis, the relationship and contribution of these lineages are unclear.

Transgenic murine models permit the spatial and temporal labeling of distinct cell populations for lineage tracing and functional studies. Recent work using an alpha smooth muscle actin Cre reporter system $(\alpha$ SMA-CreERT2; R26RtdTomato) showed that SMA-labeled cells from the paratenon or perivascular regions are the main responders to a fulllength, central defect in the patellar tendon [93・•]. These cells were discovered to form a collagenous bridge over the anterior surface of the defect while also infiltrating the adjacent tendon struts, leading to hypercellular and disorganized tissue in these regions. These cells also are proliferative and express tendon markers, including Scx-GFP, Fibromodulin, and Tenascin C $[6,93 \bullet \cdot]$. Consistent with these findings, increased tendon growth in a different injury model appears to arise from the superficial surrounding layers of the tendon [94•]. These observations may be comparable to the response of the periosteum in bone fracture healing, where the surrounding periosteal cells can be activated following injury to form chondrocytes in the fracture callus and osteoblasts in the healed bone [95-98] Together, these studies point towards an extrinsic cell population from the surrounding connective tissue that activates following injury to contribute to the repair response. However, it is unclear from these data if this is the only responding cell population, and several scenarios are still possible. The extrinsic population could function similar to a stem or progenitor cell model where relatively quiescent cells are activated to reconstitute the tissue following injury. Alternatively, intrinsic and extrinsic cells may also contribute at different stages to the long-term repair of the tissue. In addition, it has not been tested whether a sub-population of mesenchymal cells, that do not turn on $S c x$ or other tendon markers, instead contribute to fibrosis as in heart and kidney models [99]. Lineage tracing studies using multispectral clonal labeling and the development of tendon or pericyte cell-specific Cre lines are necessary to better delineate the specific cell contributions to tendon healing in both acute and chronic injury models.

\section{Regenerative Model Systems}

As the musculoskeletal field transitions from reparative to regenerative medicine, researchers are attempting to gain insight from animal models that display either improved healing or true regenerative capabilities. These models include the salamander (e.g., axolotl and newt), zebrafish, and Murphy Roths Large (MRL) mouse. Zebrafish and axolotls share similar modes of regulation of their musculoskeletal tissues with humans [100-103] and have very robust regenerative potential. The MRL mouse is the parent and control strain for MRL/ MpJ-Fas ${ }^{\text {lpr }}$ mouse, which has systemic lupus erythematosus and Sjorgren syndrome [104]. These mice display improved healing capacity in some, but not all, injury models [105-112]. Investigators hope that their discoveries in these systems can be applied to humans to improve repair outcomes and achieve true regeneration. From these models several key unifying concepts have begun to emerge that have the potential to guide tendon repair strategies in humans.

Examination of epimorphic regeneration in salamanders has led to a greater understanding of cell-specific lineage relationships during the regenerative process. An adult axolotl can entirely re-grow an amputated limb, and this process begins with epithelial closure of the wound site and formation of 
a blastema [113-116]. The blastema is composed of undifferentiated cells that will give rise to the newly formed limb tissues. Although these cells were once thought to be homogeneous in nature, a pivotal study by Kragl et al. demonstrated that cells within the blastema retain a memory of their tissue of origin. The blastema cells are restricted in their ability to contribute to new tissues and limb regeneration does not involve dedifferentiation of cells to a pluripotent state [113]. Lineage restriction was also observed in the zebrafish and mammalian models using similar lineage tracing strategies $[46,117,118]$. Studies examining endogenous mesenchymal populations in mouse bone marrow also discovered lineage restriction in vivo compared with their in vitro potential [119]. Although there are major differences between tendon and bone injury repair and epimorphic limb regeneration, these studies highlight how lineage relationships can impact our understanding of progenitor cell potential. Furthermore, understanding the cues that direct regeneration of a new functioning tendon tissue from undifferentiated progenitor cells would be useful for tissue engineering based regenerative approaches.

Work in regenerative organisms also points to participation from multiple cell types in the regenerative process. In particular, inflammatory cells have been shown in multiple systems to be important for repair or regeneration to occur. In axolotl, depletion of macrophages and other phagocytic cells will disrupt regeneration after the wound closure stages, resulting in fibrosis and aberrant gene expression in the failed stump [114]. In addition, macrophages were found to actively clear senescent cells from normal and regenerating salamander limbs, suggesting an important role for these cells in maintaining regenerative potential [120]. A role for macrophages has also been described for zebrafish tailfin regeneration [121]. However, macrophages have been implicated in having a detrimental role in mammalian tissue repair and in contributing towards fibrosis [122]. In fact, a reduced inflammatory response may contribute towards improved healing outcomes in MRL mouse injury models [123, 124]. The mechanism contributing towards their improved healing response is not well understood, although genetic mapping has identified cell cycle regulators $[125,126]$. Tendon injuries in MRL mice have an improved healing response with increased mechanical properties and cell proliferation and a reduction in the macrophage response [124]. Distinct macrophage populations have been shown to infiltrate mammalian tendon tissue at specific stages after injury along with differential regulation of pro-inflammatory cytokines [127]. In addition, much work has been done examining the role of cytokines in tendon cell behaviors [128]. It is likely that macrophages and the inflammatory response is necessary for clearance of dead or dying tissues, but prolonged activity and secretion of cytokines could result in negative fibrotic or degenerative effects. In order to define the specific roles of the macrophage populations, temporally and spatially specific loss of function studies are necessary during normal, injured, and aged conditions. It is also possible that the cell proliferation changes may play a role in the differences that were observed. Future work with the MRL mouse model will expand our understanding of improved tendon healing responses in a mammalian genetic system.

The zebrafish is another regenerative model system that has garnered a great deal of attention for its ability to robustly regenerate characteristically non-regenerative tissues such as the heart and nephrons of the kidney $[129,130]$. Manipulation of pathways discovered in zebrafish cardiac regeneration have been found to improve heart functionality after injury in mice, demonstrating that knowledge gained from fish can impact mammalian injury outcomes [131]. Recent work has begun to describe zebrafish tendons $[102,132]$, and future work is aimed at understanding the regenerative potential of their tendon tissues. The ability to perform functional and live imaging studies in the zebrafish along with advances in axolotl genetic manipulations and the MRL mouse model $[133,134]$ will undoubtedly lead to new knowledge of the basic mechanisms underlying tendon regenerative biology.

\section{Conclusion}

As researchers continue to develop better in vivo genetic models for identifying resident progenitor cells in tendons, the next step will be to understand the manner by which these cells contribute to tendon development, growth, and repair (Fig. 1). It will be important to determine if the resident progenitors are situated in specific regions of the tendon and subject to regulation by their surrounding environment or niche. It will also be essential to understand the specific pathways and additional cell types that regulate their behaviors during changes in physical activity and aging. Many of these questions can be answered with detailed lineage tracing analysis and conditional knockouts of key functional genes. Comparisons between regenerative and nonregenerative model systems will also provide new perspectives on repair mechanisms and candidate pathways to test in improving injury outcomes. By performing these studies in vivo with genetic and regenerative model systems, we will gain valuable information about the endogenous phenotypes of these resident populations and provide key design criteria for future therapeutic strategies.

Acknowledgments The authors would like to acknowledge the following funding sources: NIH/NIDCR 5T90DE021989 (NAD), NIH/NICHD 5R00HD069533 (JLG), and a seed grant from the Harvard Stem Cell Institute (JLG).

\section{Compliance with Ethics Guidelines}

Conflict of Interest Nathaniel A. Dyment and Jenna L. Galloway declare that they have no conflict of interest. 
Human and Animal Rights and Informed Consent This article does not contain any studies with human subjects performed by any of the authors. With regard to the authors' research cited in this paper, all institutional and national guidelines for the care and use of laboratory animals were followed.

\section{References}

Papers of particular interest, published recently, have been highlighted as:

- Of importance

•- Of major importance

1. Greenlee Jr TK, Beckham C, Pike D. A fine structural study of the development of the chick flexor digital tendon: a model for synovial sheathed tendon healing. Am J Anat. 1975;143(3):303-13.

2. Liu CF et al. Spatial and temporal expression of molecular markers and cell signals during normal development of the mouse patellar tendon. Tissue Eng A. 2012;18(5-6):598-608.

3. Gomoll $\mathrm{AH}$ et al. Rotator cuff disorders: recognition and management among patients with shoulder pain. Arthritis Rheum. 2004;50(12):3751-61.

4. Sharma P, Maffulli N. Basic biology of tendon injury and healing. Surgeon. 2005;3(5):309-16.

5. Dyment NA et al. The relationships among spatiotemporal collagen gene expression, histology, and biomechanics following fulllength injury in the murine patellar tendon. J Orthop Res. 2012;30(1):28-36.

6. Dyment NA et al. The paratenon contributes to scleraxisexpressing cells during patellar tendon healing. PLoS One. 2013;8(3):e59944.

7. Galatz LM et al. Tendon regeneration and scar formation: the concept of scarless healing. J Orthop Res. 2015;33(6):823-31.

8. Shadwick RE. Elastic energy storage in tendons: mechanical differences related to function and age. J Appl Physiol (1985). 1990;68(3):1033-40.

9. Arnesen SM, Lawson MA. Age-related changes in focal adhesions lead to altered cell behavior in tendon fibroblasts. Mech Ageing Dev. 2006;127(9):726-32.

10. Dressler MR et al. A potential mechanism for age-related declines in patellar tendon biomechanics. J Orthop Res. 2002;20(6):131522.

11.• Dunkman AA et al. Decorin expression is important for agerelated changes in tendon structure and mechanical properties. Matrix Biol. 2013;32(1):3-13. This paper showed that aged tendons exhibited significantly decreased viscoelastic properties with reduced cell number and collagen fiber alignment compared to mature tendons, which may help explain higher susceptibility to tendon injury in the aging population.

12. Noden DM. The role of the neural crest in patterning of avian cranial skeletal, connective, and muscle tissues. Dev Biol. 1983;96(1):144-65.

13. Brent AE, Schweitzer R, Tabin CJ. A somitic compartment of tendon progenitors. Cell. 2003;113(2):235-48.

14. Kieny M, Chevallier A. Autonomy of tendon development in the embryonic chick wing. J Embryol Exp Morphol. 1979;49:153-65.

15. Murchison ND et al. Regulation of tendon differentiation by scleraxis distinguishes force-transmitting tendons from muscleanchoring tendons. Development. 2007;134(14):2697-708.

16. Anderson DM et al. Mohawk is a novel homeobox gene expressed in the developing mouse embryo. Dev Dyn. 2006;235(3):792801 .
17. Ito $\mathrm{Y}$ et al. The Mohawk homeobox gene is a critical regulator of tendon differentiation. Proc Natl Acad Sci U S A. 2010;107(23): 10538-42.

18. Shukunami C, Oshima Y, Hiraki Y. Molecular cloning of tenomodulin, a novel chondromodulin-I related gene. Biochem Biophys Res Commun. 2001;280(5):1323-7.

19. Docheva $\mathrm{D}$ et al. Tenomodulin is necessary for tenocyte proliferation and tendon maturation. Mol Cell Biol. 2005;25(2):699-705.

20. Lejard V et al. EGR1 and EGR2 involvement in vertebrate tendon differentiation. J Biol Chem. 2011;286(7):5855-67.

21. Birk DE et al. Collagen fibrillogenesis in situ: fibril segments become long fibrils as the developing tendon matures. Dev Dyn. 1997;208(3):291-8.

22. Ezura $Y$ et al. Differential expression of lumican and fibromodulin regulate collagen fibrillogenesis in developing mouse tendons. J Cell Biol. 2000;151(4):779-88.

23. Zhang $\mathrm{G}$ et al. Development of tendon structure and function: regulation of collagen fibrillogenesis. J Musculoskelet Neuronal Interact. 2005;5(1):5-21.

24. Kalson NS, et al. A structure-based extracellular matrix expansion mechanism of fibrous tissue growth. Elife. 2015;4. Using 3Delectron miscroscopy, the authors describe that postnatal tail tendon growth is achieved through expansion of the collagen matrix. The tendon cells also change morphology as the animal matures, while maintaining connections with neighboring cells. It would be exciting to apply such powerful techniques to other tendons in the body and in injury and aging conditions.

25. Ippolito $\mathrm{E}$ et al. Morphological, immunochemical, and biochemical study of rabbit Achilles tendon at various ages. J Bone Joint Surg Am. 1980;62(4):583-98.

26. Kannus P. Structure of the tendon connective tissue. Scand J Med Sci Sports. 2000;10(6):312-20.

27. Jozsa L, Kannus P. Histopathological findings in spontaneous tendon ruptures. Scand J Med Sci Sports. 1997;7(2):113-8.

28. Davidson CJ et al. Rat tendon morphologic and functional changes resulting from soft tissue mobilization. Med Sci Sports Exerc. 1997;29(3):313-9.

29. Chuen FS et al. Immunohistochemical characterization of cells in adult human patellar tendons. J Histochem Cytochem. 2004;52(9):1151-7.

30. Banes AJ et al. Cell populations of tendon: a simplified method for isolation of synovial cells and internal fibroblasts: confirmation of origin and biologic properties. J Orthop Res. 1988;6(1):83-94.

31. Corps AN et al. The regulation of aggrecanase ADAMTS-4 expression in human Achilles tendon and tendon-derived cells. Matrix Biol. 2008;27(5):393-401.

32. Nourissat G, Berenbaum F, Duprez D. Tendon injury: from biology to tendon repair. Nat Rev Rheumatol. 2015;11(4):223-33.

33. Babraj JA et al. Collagen synthesis in human musculoskeletal tissues and skin. Am J Physiol Endocrinol Metab. 2005;289(5): E864-9.

34. Miller BF et al. Coordinated collagen and muscle protein synthesis in human patella tendon and quadriceps muscle after exercise. J Physiol. 2005;567(Pt 3):1021-33.

35. Thorpe $\mathrm{CT}$ et al. Aspartic acid racemization and collagen degradation markers reveal an accumulation of damage in tendon collagen that is enhanced with aging. J Biol Chem. 2010;285(21): 15674-81.

36. • Heinemeier KM et al. Lack of tissue renewal in human adult Achilles tendon is revealed by nuclear bomb (14)C. FASEB J. 2013;27(5):2074-9. Through ${ }^{14} \mathbf{C}$ labeling, this paper demonstrated that the bulk tendon mass was primarily generated during growth and minimal turnover occurred in adulthood.

37. Messier B, Leblond CP. Cell proliferation and migration as revealed by radioautography after injection of thymidine-H3 into male rats and mice. Am J Anat. 1960;106:247-85. 
38. Ruchti $\mathrm{C}$ et al. Regional differences in renewal rates of fibroblasts in young adult female mice. Cell Tissue Res. 1983;232(3):625-36.

39. Runesson E et al. Detection of slow-cycling and stem/progenitor cells in different regions of rat Achilles tendon: response to treadmill exercise. Knee Surg Sports Traumatol Arthrosc. 2013;21(7): 1694-703.

40. Buczacki SJ et al. Intestinal label-retaining cells are secretory precursors expressing Lgr5. Nature. 2013;495(7439):65-9.

41. Falkowska-Hansen B et al. An inducible Tet-Off-H2B-GFP lentiviral reporter vector for detection and in vivo isolation of label-retaining cells. Exp Cell Res. 2010;316(11):1885-95.

42. Mascre $\mathrm{G}$ et al. Distinct contribution of stem and progenitor cells to epidermal maintenance. Nature. 2012;489(7415):257-62.

43. Foudi A et al. Analysis of histone 2B-GFP retention reveals slowly cycling hematopoietic stem cells. Nat Biotechnol. 2009;27(1): 84-90.

44. Snippert HJ et al. Intestinal crypt homeostasis results from neutral competition between symmetrically dividing Lgr5 stem cells. Cell. 2010;143(1):134-44.

45. Schepers AG et al. Lineage tracing reveals Lgr5+ stem cell activity in mouse intestinal adenomas. Science. 2012;337(6095):730 5 .

46. Rinkevich Y et al. Germ-layer and lineage-restricted stem/ progenitors regenerate the mouse digit tip. Nature. 2011;476(7361):409-13.

47. Maeda $\mathrm{T}$ et al. Conversion of mechanical force into TGF-betamediated biochemical signals. Curr Biol. 2011;21(11):933-41.

48. Lavagnino $\mathrm{M}$ et al. Tendon mechanobiology: current knowledge and future research opportunities. J Orthop Res. 2015;33(6):81322.

49. Mendias CL et al. Physiological loading of tendons induces scleraxis expression in epitenon fibroblasts. J Orthop Res. 2012;30(4):606-12.

50. Magnusson SP, Kjaer M. Region-specific differences in Achilles tendon cross-sectional area in runners and non-runners. Eur J Appl Physiol. 2003;90(5-6):549-53.

51. Kjaer M et al. Metabolic activity and collagen turnover in human tendon in response to physical activity. J Musculoskelet Neuronal Interact. 2005;5(1):41-52.

52. Majima $\mathrm{T}$ et al. Biomechanical effects of stress shielding of the rabbit patellar tendon depend on the degree of stress reduction. J Orthop Res. 1996;14(3):377-83

53. Maeda E et al. Differential regulation of gene expression in isolated tendon fascicles exposed to cyclic tensile strain in vitro. J Appl Physiol (1985). 2009;106(2):506-12.

54. Carpenter JE et al. The effects of overuse combined with intrinsic or extrinsic alterations in an animal model of rotator cuff tendinosis. Am J Sports Med. 1998;26(6):801-7.

55. Riley G. Chronic tendon pathology: molecular basis and therapeutic implications. Expert Rev Mol Med. 2005;7(5):1-25.

56. Attia $\mathrm{M}$ et al. Alterations of overused supraspinatus tendon: a possible role of glycosaminoglycans and HARP/pleiotrophin in early tendon pathology. J Orthop Res. 2012;30(1):61-71.

57. Samiric $\mathrm{T}$ et al. Changes in the composition of the extracellular matrix in patellar tendinopathy. Matrix Biol. 2009;28(4):230-6.

58. Parkinson $\mathrm{J}$ et al. Change in proteoglycan metabolism is a characteristic of human patellar tendinopathy. Arthritis Rheum. 2010;62(10):3028-35.

59. Archambault JM et al. Rat supraspinatus tendon expresses cartilage markers with overuse. J Orthop Res. 2007;25(5):617-24.

60. Scott $\mathrm{A}$ et al. Increased versican content is associated with tendinosis pathology in the patellar tendon of athletes with jumper's knee. Scand J Med Sci Sports. 2008;18(4):427-35.

61. Goh KL et al. Ageing changes in the tensile properties of tendons: influence of collagen fibril volume fraction. J Biomech Eng. 2008;130(2):021011.
62. Yamamoto $\mathrm{Z}$ et al. Effect of suture repair on expression of beta1 integrin subunit in wounded rat patellar tendon. J Orthop Sci. 2004;9(6):613-8.

63. Plate JF et al. Advanced age diminishes tendon-to-bone healing in a rat model of rotator cuff repair. Am J Sports Med. 2014;42(4): 859-68.

64. Durant TJ et al. Mesenchymal stem cell response to growth factor treatment and low oxygen tension in 3-dimensional construct environment. Muscles Ligaments Tendons J. 2014;4(1):46-51.

65. Waugh $\mathrm{CM}$ et al. Age-related changes in mechanical properties of the Achilles tendon. J Anat. 2012;220(2):144-55.

66. Stenroth L et al. Age-related differences in Achilles tendon properties and triceps surae muscle architecture in vivo. J Appl Physiol (1985). 2012;113(10):1537-44.

67. Trindade VL et al. Experimental study of the influence of senescence in the biomechanical properties of the temporal tendon and deep temporal fascia based on uniaxial tension tests. J Biomech. 2012;45(1):199-201.

68. Zhou $Z$ et al. Tendon-derived stem/progenitor cell aging: defective self-renewal and altered fate. Aging Cell. 2010;9(5):911-5.

69. Lopez-Otin $\mathrm{C}$ et al. The hallmarks of aging. Cell. 2013;153(6): 1194-217.

70. Kubo K et al. Relationship between Achilles tendon properties and foot strike patterns in long-distance runners. J Sports Sci. 2015;33(7):665-9.

71. Peffers MJ et al. Proteomic analysis reveals age-related changes in tendon matrix composition, with age- and injury-specific matrix fragmentation. J Biol Chem. 2014;289(37):25867-78.

72. Kohler $\mathrm{J}$ et al. Uncovering the cellular and molecular changes in tendon stem/progenitor cells attributed to tendon aging and degeneration. Aging Cell. 2013;12(6):988-99.

73. Chen L et al. miR-135a modulates tendon stem/progenitor cell senescence via suppressing ROCK1. Bone. 2015;71:210-6.

74. Snippert HJ et al. Lgr6 marks stem cells in the hair follicle that generate all cell lineages of the skin. Science. 2010;327(5971): 1385-9.

75. Blanpain C, Fuchs E. Stem cell plasticity. Plasticity of epithelial stem cells in tissue regeneration. Science. 2014;344(6189): 1242281 .

76. Stanger BZ, Hebrok M. Control of cell identity in pancreas development and regeneration. Gastroenterology. 2013;144(6):1170-9.

77. Schaub JR et al. Evidence against a stem cell origin of new hepatocytes in a common mouse model of chronic liver injury. Cell Rep. 2014;8(4):933-9.

78. Senyo SE, Lee RT, Kuhn B. Cardiac regeneration based on mechanisms of cardiomyocyte proliferation and differentiation. Stem Cell Res. 2014;13(3 Pt B):532-41.

79. Leask A. Getting to the heart of the matter: new insights into cardiac fibrosis. Circ Res. 2015;116(7):1269-76.

80. Rockey DC, Bell PD, Hill JA. Fibrosis - a common pathway to organ injury and failure. N Engl J Med. 2015;372(12):1138-49.

81. Bi Y et al. Identification of tendon stem/progenitor cells and the role of the extracellular matrix in their niche. Nat Med. 2007;13(10):1219-27.

82. Ni M et al. Tendon-derived stem cells (TDSCs) promote tendon repair in a rat patellar tendon window defect model. J Orthop Res. 2012;30(4):613-9.

83. Gelberman RH et al. Fibroblast chemotaxis after tendon repair. J Hand Surg [Am]. 1991;16(4):686-93.

84. Lundborg G, Rank F. Experimental intrinsic healing of flexor tendons based upon synovial fluid nutrition. J Hand Surg [Am]. 1978;3(1):21-31.

85. Manske PR et al. Intrinsic flexor-tendon repair. A morphological study in vitro. J Bone Joint Surg Am. 1984;66(3):385-96. 
86. Matthews P, Richards H. Factors in the adherence of flexor tendon after repair: an experimental study in the rabbit. J Bone Joint Surg (Br). 1976;58(2):230-6.

87. Harrison RK et al. Synovial sheath cell migratory response to flexor tendon injury: an experimental study in rats. J Hand Surg [Am]. 2003;28(6):987-93.

88. Potenza AD. Tendon healing within the flexor digital sheath in the dog. J Bone Joint Surg Am. 1962;44-A:49-64.

89. Skoog T, Persson BH. Plast Reconstr Surg (1946). 1954;13(5): 384-99.

90. Branford OA et al. The mechanics of flexor tendon adhesions. J Hand Surg Eur Vol. 2012;37(6):555-63.

91. Tempfer $\mathrm{H}$ et al. Perivascular cells of the supraspinatus tendon express both tendon- and stem cell-related markers. Histochem Cell Biol. 2009;131(6):733-41.

92. Beredjiklian PK. Biologic aspects of flexor tendon laceration and repair. J Bone Joint Surg Am. 2003;85-A(3):539-50.

93.•- Dyment NA et al. Lineage tracing of resident tendon progenitor cells during growth and natural healing. PLoS One. 2014;9(4): e96113. This paper demonstrated that SMA-labeled paratenon and/or perivascular cells contribute to Scxexpressing cells during tendon healing.

94. Gumucio JP et al. Synergist ablation induces rapid tendon growth through the synthesis of a neotendon matrix. J Appl Physiol (1985). 2014;117(11):1287-91. The authors use a synergist ablation model to show that new tendon growth appears to arise from the surrounding layers of the tendon while the internal tendon cells appear to be postmitotic.

95. Colnot C. Skeletal cell fate decisions within periosteum and bone marrow during bone regeneration. J Bone Miner Res. 2009;24(2): 274-82.

96. Murao $\mathrm{H}$ et al. Periosteal cells are a major source of soft callus in bone fracture. J Bone Miner Metab. 2013;31(4):390-8.

97. Grcevic D et al. In vivo fate mapping identifies mesenchymal progenitor cells. Stem Cells. 2012;30(2):187-96.

98. Matthews BG et al. Analysis of alphaSMA-labeled progenitor cell commitment identifies notch signaling as an important pathway in fracture healing. J Bone Miner Res. 2014;29(5):1283-94.

99. Kramann R et al. Perivascular Gli1+ progenitors are key contributors to injury-induced organ fibrosis. Cell Stem Cell. 2015;16(1): 51-66.

100. Cosden-Decker RS et al. Structural and functional analysis of intra-articular interzone tissue in axolotl salamanders. Osteoarthr Cartil. 2012;20(11):1347-56.

101. Bruneel B, Witten PE. Power and challenges of using zebrafish as a model for skeletal tissue imaging. Connect Tissue Res. 2015;56(2):161-73.

102. Chen JW, Galloway JL. The development of zebrafish tendon and ligament progenitors. Development. 2014;141(10):2035-45.

103. Rossi G, Messina G. Comparative myogenesis in teleosts and mammals. Cell Mol Life Sci. 2014;71(16):3081-99.

104. Mountz JD, Gause WC, Jonsson R. Murine models for systemic lupus erythematosus and Sjogren's syndrome. Curr Opin Rheumatol. 1991;3(5):738-56.

105. Clark LD, Clark RK, Heber-Katz E. A new murine model for mammalian wound repair and regeneration. Clin Immunol Immunopathol. 1998;88(1):35-45.

106. Rai MF et al. Heritability of articular cartilage regeneration and its association with ear wound healing in mice. Arthritis Rheum. 2012;64(7):2300-10

107. Lewis Jr JS et al. Genetic and cellular evidence of decreased inflammation associated with reduced incidence of posttraumatic arthritis in MRL/MpJ mice. Arthritis Rheum. 2013;65(3):660-70.

108. Thuret $\mathrm{S}$ et al. Enhanced functional recovery in $\mathrm{MRL} / \mathrm{MpJ}$ mice after spinal cord dorsal hemisection. PLoS One. 2012;7(2): e30904.
109. Grisel P et al. The MRL mouse repairs both cryogenic and ischemic myocardial infarcts with scar. Cardiovasc Pathol. 2008;17(1): $14-22$.

110. Turner NJ, Johnson SA, Badylak SF. A histomorphologic study of the normal healing response following digit amputation in $\mathrm{C} 57 \mathrm{bl} / 6$ and MRL/MpJ mice. Arch Histol Cytol. 2010;73(2):103-11.

111. Davis TA et al. Differential cutaneous wound healing in thermally injured MRL/MPJ mice. Wound Repair Regen. 2007;15(4):577-88.

112. Beare AH, Metcalfe AD, Ferguson MW. Location of injury influences the mechanisms of both regeneration and repair within the MRL/MpJ mouse. J Anat. 2006;209(4):547-59.

113. Kragl $\mathrm{M}$ et al. Cells keep a memory of their tissue origin during axolotl limb regeneration. Nature. 2009;460(7251):60-5.

114. Godwin JW, Pinto AR, Rosenthal NA. Macrophages are required for adult salamander limb regeneration. Proc Natl Acad Sci U S A. 2013;110(23):9415-20.

115. Kumar A, Gates PB, Brockes JP. Positional identity of adult stem cells in salamander limb regeneration. C R Biol. 2007;330(6-7):485-90.

116. Sahenk Z, Oblinger J, Edwards C. Neurotrophin-3 deficient Schwann cells impair nerve regeneration. Exp Neurol. 2008;212(2):552-6.

117. Lehoczky JA, Robert B, Tabin CJ. Mouse digit tip regeneration is mediated by fate-restricted progenitor cells. Proc Natl Acad Sci U S A. 2011;108(51):20609-14.

118. Tu S, Johnson SL. Fate restriction in the growing and regenerating zebrafish fin. Dev Cell. 2011;20(5):725-32.

119. Park D et al. Endogenous bone marrow MSCs are dynamic, faterestricted participants in bone maintenance and regeneration. Cell Stem Cell. 2012;10(3):259-72.

120. Yun MH, Davaapil H, Brockes JP. Recurrent turnover of senescent cells during regeneration of a complex structure. Elife. 2015. 4.

121. Petrie TA et al. Macrophages modulate adult zebrafish tail fin regeneration. Development. 2014;141(13):2581-91.

122. Tacke F, Zimmermann HW. Macrophage heterogeneity in liver injury and fibrosis. J Hepatol. 2014;60(5):1090-6.

123. Ueno $\mathrm{M}$ et al. Accelerated wound healing of alkali-burned corneas in MRL mice is associated with a reduced inflammatory signature. Invest Ophthalmol Vis Sci. 2005;46(11):4097-106.

124. Lalley AL, et al. Improved biomechanical and biological outcomes in the MRL/MpJ murine strain following a full-length patellar tendon injury. J Orthop Res. 2015.

125. Bedelbaeva $\mathrm{K}$ et al. Lack of $\mathrm{p} 21$ expression links cell cycle control and appendage regeneration in mice. Proc Natl Acad Sci U S A. 2010;107(13):5845-50.

126. Arthur LM, Heber-Katz E. The role of p21 in regulating mammalian regeneration. Stem Cell Res Ther. 2011;2(3):30.

127. Sugg KB et al. Changes in macrophage phenotype and induction of epithelial-to-mesenchymal transition genes following acute Achilles tenotomy and repair. J Orthop Res. 2014;32(7):944-51.

128. Schulze-Tanzil $\mathrm{G}$ et al. The role of pro-inflammatory and immunoregulatory cytokines in tendon healing and rupture: new insights. Scand J Med Sci Sports. 2011;21(3):337-51.

129. Poss KD, Wilson LG, Keating MT. Heart regeneration in zebrafish. Science. 2002;298(5601):2188-90.

130. Diep CQ et al. Identification of adult nephron progenitors capable of kidney regeneration in zebrafish. Nature. 2011;470(7332):95-100.

131. Aguirre $\mathrm{A}$ et al. In vivo activation of a conserved microRNA program induces mammalian heart regeneration. Cell Stem Cell. 2014;15(5):589-604.

132. Shah RR et al. Tensile properties of craniofacial tendons in the mature and aged zebrafish. J Orthop Res. 2015;33(6):867-73.

133. Whited JL et al. Pseudotyped retroviruses for infecting axolotl in vivo and in vitro. Development. 2013;140(5):1137-46.

134. Flowers GP et al. Highly efficient targeted mutagenesis in axolotl using Cas9 RNA-guided nuclease. Development. 2014;141(10): 2165-71. 